\title{
Desafíos de las Políticas Educativas frente a la Formación Inicial y Desarrollo Profesional Docente ${ }^{1}$
}

\section{Challenges of the Educational Policies for initial training and Teacher's profesional development}

Iván José Medina Payarez²

\section{Resumen}

El objetivo de este trabajo es presentar los principales desafíos de las políticas educativas relacionadas con la formación inicial de los educadores y algunos elementos claves de los sistemas de actualización y desarrollo profesional de docentes en ejercicio. Frente a la formación inicial se precisa la importancia de lograr un equilibrio entre la formación disciplinaria y el dominio de estrategias didácticas y pedagógicas para impartir su enseñanza, es decir, avanzar en el fortalecimiento de los diseños curriculares de los programas de formación docente teniendo como foco de atención el desarrollo del conocimiento didáctico del contenido. Con respecto al sistema de actualización y desarrollo profesional de educadores en servicio se resalta su complejidad, teniendo en cuenta, la diversidad de perfiles de los profesores y la gran variedad de contextos donde se promueven los procesos de enseñanza aprendizaje. En este sentido, se valora la pertinencia del acompañamiento pedagógico de los educadores en ejercicio considerando el análisis sobre la planeación y la puesta en escena de los procesos de intervención docente mediante la consolidación de comunidades de aprendizaje.

Palabras clave: Formación; Docente; Didáctica;

Políticas educativas; Currículo.
The objective of this paper is to present the main challenges of the educational policies related to the initial training of educators, and some key elements of the updating and professional development systems for teachers in service. About initial training, it is necessary to achieve a balance between disciplinary training and mastery of didactic and pedagogical strategies for their teaching, that is, to advance in the strengthening of curricular designs of teacher training programs focusing on the development of didactic knowledge of content. With regard to the systems of updating and professional development of in-service educators, the complexity of the system is emphasized, taking into account the diversity of teacher profiles and the wide variety of contexts where teaching-learning processes are promoted. In this sense, the relevance of the pedagogical accompaniment of the educators in exercise is valued considering the analysis of planning and staging of the processes of teacher intervention through the consolidation of learning communities.

Keywords: Training; Teacher; Didactics; Educational policies; Curriculum. 


\section{Introducción}

En este artículo se reflexiona en torno a los principales retos y desafíos de las políticas educativas relacionadas con la formación inicial docente, y con los sistemas de actualización y desarrollo profesional de educadores en servicio. Para el análisis se consideran algunos cambios que se han realizado en el contexto de su aplicación y que son fundamentales para comprender el rumbo que han seguido en los últimos años.

En el plano de la formación inicial docente se presenta un debate sobre la profesionalización docente desde el currículo de las universidades y de las escuelas normales como instituciones encargadas de la formación de los estudiantes de docencia. Entre los hallazgos se determina que los docentes de primaria "deben tener una mejor formación en lo referente a las disciplinas del curriculum, mientras que los docentes de media necesitan ser formados en todo lo referente a las cuestiones pedagógicas para el abordaje en el aula de las disciplinas" (Martin, 1999, citado en Aguerrondo, 2003, p.108). De igual forma con relación a los maestros del nivel de educación media Pogré y Merodo (2006) sostienen que "se ha logrado una alta especialización en las disciplinas con un escaso peso en la formación pedagógica” (p.64); mientras que los educadores del nivel de primaria demuestran fortalezas en las teorías y enfoques pedagógicos, pero con bajo dominio disciplinario.

Se establece como reto para los sistemas de formación inicial docente el diseño de un currículo que posibilite que el educador se empodere de los contenidos de la disciplina que enseña y de igual forma logre un dominio de estrategias pedagógicas y didácticas que favorezcan los procesos de formación integral de los estudiantes. Esto significa afianzar el conocimiento didáctico del contenido, es decir, profundizar tanto en las estructuras teóricas del objeto de estudio como en la exploración de las formas más apropiadas para impartir su enseñanza, y promover los aprendizajes de los estudiantes en forma significativa.

Con respecto a los procesos de actualización y desarrollo profesional de los docentes en ejercicio es pertinente tener en cuenta la complejidad del diseño de las políticas de formación docente y las razones por las cuales se reconoce su incidencia como programa que apunta al mejoramiento de la calidad de la educación. Este proceso es complejo atendiendo a la diversidad de perfiles de los equipos docentes que conforman el sistema educativo, y de igual forma por los contextos diversos en que se desenvuelven los educadores atendiendo las diferentes problemáticas y necesidades de los entornos en los que se movilizan los estudiantes.

En el marco de las estrategias de actualización y desarrollo profesional docente se subraya el acompañamiento pedagógico en el que se generan nuevos escenarios de reflexión entre pares académicos en torno a procesos de planeación y puesta en escena de las clases. En este sentido, el reto de las políticas educativas asociadas a la formación docente es consolidar un sistema educativo que permita afianzar las comunidades de aprendizaje mediante el trabajo cooperativo aprovechando las fortalezas de los docentes como actores fundamentales en esta apuesta.

\section{Consideraciones teóricas}

Las consideraciones teóricas del artículo están organizadas atendiendo a los principales desafíos identificados en las políticas públicas de formación docente en dos categorías. Por una parte, las reflexiones teóricas relacionadas con la profesionalización o formación inicial de los docentes, y por otra los referentes teóricos que permiten el análisis de los procesos de actualización y desarrollo profesional de los educadores en ejercicio. 
Desafíos de las Políticas Educativas frente a la Formación inicial y Desarrollo Profesional Docente

Frente a los procesos de formación docente se propone una reflexión teórica en torno a los problemas del nivel curricular que enfrentan las universidades y las escuelas normales en el cumplimiento de la responsabilidad social de formar a los futuros educadores y se identifican los principales desafíos y retos en la apuesta por mejorar la calidad de los mismos. Así mismo, se plantean elementos teóricos para el análisis de las políticas de actualización y desarrollo profesional de los educadores en ejercicio caracterizando las oportunidades de mejora a partir de los retos identificados en esta área.

\section{Profesionalización y formación inicial docente}

La profesionalización y formación inicial de los educadores se constituye en uno de los temas de mayor relevancia en las agendas de política pública de los estados en su intención de mejorar la calidad de la educación. En este análisis se abordan diferentes perspectivas sobre el desarrollo de los procesos de profesionalización de los docentes, $\mathrm{y}$ algunos de los cambios que se han promovido desde el ámbito curricular en las instituciones que se encargan de formarlos.

Ahora bien, frente a la formación inicial de los docentes Imbernon (1989) sostiene que "ha sido insuficiente para suministrar a los futuros profesores los conocimientos, generales y específicos, y los principios psicopedagógicos y científicos necesarios para desarrollar posteriormente una buena tarea profesional" (p.488). Así mismo, se ha reiterado en el escenario latinoamericano en el que se evidencian importantes proyectos de reforma en la formación inicial de los docentes, a tal punto que se han establecido como condiciones, la acreditación de calidad de los programas de formación profesional vinculadas a la actividad docente en las facultades de educación de las universidades, y se ha llegado a cuestionar incluso la tarea de las Escuelas Normales como instituciones que tienen la misión, en varios países latinoamericanos, de formar a los futuros maestros y maestras.

Es significativo enfocar la reflexión en el tema de la formación inicial de los docentes en la medida en que se define como "un trayecto donde se introducen al conocimiento sistematizado de disciplinas asociadas con la educación y, parcialmente, a la práctica escolar y a la de enseñanza" (Mercado, 2010, p.151). De igual forma se reconoce que:

Las nuevas demandas sociales hacia la educación han originado la necesidad de profundos cambios no solo en relación a los contenidos que se enseñan, o con los modelos didácticos con que se trabajan en las escuelas, sino también en relación a una redefinición de la tarea de enseñar $y$, por lo tanto, de la formación de los profesores. (Aguerrondo, 2003, p.105).

Esto ciertamente ha demandado una serie de transformaciones en los diseños curriculares de los programas de formación inicial de docentes en los que se cuestiona el énfasis de las facultades de educación de las universidades con relación al desarrollo de las competencias disciplinares, y se han reconocido debilidades de las mallas curriculares en la formación de estrategias didácticas para su enseñanza, y con respecto a las Escuelas Normales se han detectado deficiencias en el empoderamiento de los contenidos disciplinares, y fortalezas en el componente de formación didáctica y pedagógica. En este sentido Martin (1999) sostiene que los docentes de primaria "deben tener una mejor formación en lo referente a las disciplinas del currículo, mientras que los docentes de media necesitan ser formados en todo lo referente a las 
cuestiones pedagógicas para el abordaje en el aula de las disciplinas" (citado en Aguerrondo, 2003, p.108).

En este sentido, el reto de las políticas educativas relacionadas con la profesionalización docente es precisamente el diseño de un modelo de formación inicial que propenda por el equilibrio entre el desarrollo de los conocimientos disciplinarios y el fortalecimiento de las competencias pedagógicas y didácticas para promover los aprendizajes en forma significativa. Esto sugiere proyectar un sistema de formación inicial docente que tanga como eje central el desarrollo del conocimiento didáctico del contenido, es decir, en la planeación de un currículo que permita el diseño de estrategias didácticas que favorezcan el empoderamiento de los contenidos declarativos de las disciplinas y el aprendizaje significativo de los estudiantes.

Sobre la tensión entre lo disciplinar y lo pedagógico Murillo (2006) sostiene que:

Es frecuente que en la formación de maestros de educación infantil y primaria el eje organizador sea el proceso de adquisición de los elementos instrumentales por parte de los niños, dejando a las disciplinas como mero elemento organizador, mientras que en la formación de docentes de secundaria el peso recae en las disciplinas, relegándose la formación pedagógica a un segundo lugar, tanto en importancia como en distribución temporal. (p.29).

En virtud de esta realidad es necesario la consolidación de un sistema de formación docente inicial basado en conocimientos teóricos y científicos de las diferentes disciplinas del plan de estudios que favorezca el dominio de estrategias pedagógico-didácticas que faciliten los procesos de enseñanza aprendizaje.

En lo que concierne específicamente a la formación de docentes para el nivel de educación inicial "se privilegió el hacer cotidiano sobre la necesidad de que el maestro supiera enseñar, considerando a la vocación como una cualidad natural" (Pogré y Merodo, 2006, p.61). En este contexto, la práctica pedagógica es un componente imprescindible para el mejoramiento continuo y perfeccionamiento curricular de los programas de formación orientados por las Escuelas Normales. No obstante, se resalta que la formación de docentes para el nivel medio se ha centrado históricamente en "la especialización en las disciplinas con un escaso peso en la formación pedagógica" (Pogré y Merodo, 2006, p.64). Estas realidades exigen de la reestructuración del diseño curricular de los programas de formación de docentes, tanto del nivel inicial como del nivel medio, instaurando un equilibrio entre lo disciplinar y lo pedagógico.

En esta transformación curricular también es imperioso considerar que la realidad es dinámica y está en permanente evolución, lo cual, exige la resignificación de los ambientes educativos para hacer frente a las problemáticas sociales, a los desafíos y retos que han surgido en la complejidad misma de dichos cambios. Para lograr con pertinencia que el sistema educativo tenga en cuenta las nuevas demandas que impone el desarrollo de un mundo globalizado, es pertinente la reconfiguración de los sistemas de formación de docentes.

Ahora bien, en concordancia con lo anterior Gil (1997) sostiene que "la formación docente no corresponde con la realidad social en que se desarrollan los estudiantes. Pero es claro que las nuevas demandas inducen un cambio de perspectiva, nuevos estilos de enseñanza, en un marco de permanente revisión y cuestionamiento". (Citado en Paniagua, 2004, p.3). Estos elementos son claves para reorientar el diseño curricular de los sistemas de formación de docentes, tanto en el nivel inicial, como en los procesos de desarrollo profesional de los educadores en ejercicio.

En este sentido, los retos que han surgido en la 
formación docente desde una perspectiva pedagógica y didáctica están centrados básicamente en la:

Actualización en el campo de la propia disciplina y sus desarrollos científicos, como una evidente formación pedagógica. Esta última aparece como uno de los temas de primer orden, no para llenar a los maestros de teorías y enfoques pedagógicos para aumentar su acervo teórico, sino por los retos y posibilidades que la discusión pedagógica puede ofrecerle". (Universidad de la Salle, 2010, p.49).

Las universidades han tenido un papel histórico en la oferta de formación de docentes de nivel medio, y en la gran mayoría de los casos se reconoce en sus estructuras curriculares un fuerte énfasis en el desarrollo de conocimientos específicos de la disciplina con un alto nivel de especialización, y un débil abordaje de las teorías pedagógicas y didácticas que en la práctica faciliten la orientación de procesos de enseñanza aprendizaje sobre la base de dichos contenidos disciplinares.

Los hallazgos se configuran en evidencia para corroborar que en muchos casos los docentes del nivel de educación media tienen fortalezas en los conocimientos específicos y especializados de su disciplina de formación, y se ven enfrentados a escenarios cotidianos donde los estudiantes no captan con facilidad la filosofía y los principios de dichos contenidos, debido a que se privilegian en el aula las intervenciones expositivas del docente y se limitan las posibilidades de participación activa de los estudiantes.

En este contexto el principal desafío para las políticas educativas de los estados, y en especial para los centros encargados de promover los procesos de profesionalización de los educadores se centra en la necesidad de desarrollar competencias docentes basadas en el dominio de estrategias de la didáctica y de la pedagogía que favorezcan el rol de mediador entre los estudiantes y los saberes específicos de la disciplina que orienta, con base en necesidades del contexto y aprovechando los intereses de los estudiantes.

Las políticas de formación inicial docente requieren para su optimización la instauración de un equilibrio entre teoría y práctica. En esta búsqueda la formación In situ posibilita poner en contexto los procesos formativos del docente aproximándolo al abordaje de situaciones reales en ambientes escolares determinados.

La organización de experiencias realmente formativas situadas en la escuela ha significado un esfuerzo por elegir con cuidado los lugares escolares de trabajo para los estudiantes de pedagogía, buscando ofrecer diversidad de contextos al mismo tiempo que situaciones donde el aprendizaje docente sea posible. (Aguerrondo, 2003, p.109)

Los bajos niveles de dedicación al desarrollo de experiencias de formación situada de los diferentes programas de docencia se dimensionan como una necesidad en el ámbito latinoamericano dado que "el énfasis de la formación es teórico y poco orientado a desarrollar comportamientos y prácticas pedagógicas específicas". (Vaillant, 2013, p.194). Por su parte, Cornejo (2014) sostiene que "las prácticas permitirían a los estudiantes en formación docente una demostración de su nivel de dominio de las habilidades juzgadas como esenciales para enseñar, lo que requiere ser evaluado y establecido oficialmente". (p.241). Así mismo, Correa (2011) afirma que "las prácticas tienen una muy buena percepción en los estudiantes porque, finalmente, están en el fuego de la acción, están realmente aprendiendo a ser docentes”. (p.79). Sin embargo, es importante considerar que la formación profesional de los docentes no puede fundamentarse sólo en sabe- 
res soportados por la práctica, sino que también es necesario el estudio de los conceptos y de la teoría para confrontar su experiencia.

Lo expuesto anteriormente sugiere la existencia de un nuevo reto para las facultades de educación de las universidades y en general para las políticas educativas de formación inicial docente, teniendo en cuenta, la necesidad de avanzar en el diseño de una oferta académica en la que se establezca un equilibrio entre el estudio de las disciplinas desde lo teórico y doctrinal, y las prácticas pedagógicas en ambientes escolares reales.

Por consiguiente, es primordial en los procesos de formación docente el aprovechamiento de los escenarios escolares como entornos idóneos para el desarrollo de las prácticas pedagógicas de los educadores en formación, favoreciendo la adquisición de competencias esenciales para el ejercicio profesional de los maestros. Estas tendencias teóricas sugieren:

La orientación de prácticas docentes basadas en la conformación de significados generadores de sentido, para abrir la posibilidad de cómo se sitúa el significado en la realidad y evitar el vacío, es decir, dar cuenta de los posibles sentidos en la adaptación práctica a la vida cotidiana” (Rodríguez, 2014, p.330)

Estudios realizados en países de la región detectan que "no existen adecuados espacios y procesos de práctica y de contacto durante los estudios a la profesión, por parte de los estudiantes de docencia" (Internacional de la Educación Oficina Regional para América Latina, 2014, p.25). Este hallazgo investigativo confirma la necesidad de que los sistemas educativos, y en especial, las políticas de formación inicial de los docentes avancen en el re-direccionamiento de sus enfoques de diseño curricular, instaurando el equilibrio entre el estudio de teorías y las prácticas pedagógicas situadas como componente sustancial del itinerario de formación pedagógica.

Las perspectivas teóricas referenciadas anteriormente revalidan la importancia de la práctica como un elemento reflexivo y fundamental en la formación inicial de los educadores, posibilitando el desarrollo de saberes situados en contextos diversos y reales que le posibilitan al docente en formación fortalecer competencias esenciales para un desempeño pertinente de acuerdo con las necesidades de los entornos en los que se movilizan los estudiantes.

En síntesis, se reconocen en el contexto de los procesos de formación inicial de los docentes dos importantes retos. Por una parte, lograr que los currículos tanto de Escuelas Normales como de los programas de formación en docencia de las facultades de educación de las universidades, instauren un equilibrio entre la formación disciplinar y la formación didáctica-pedagógica; y por otra que la ruta de formación docente permita el empoderamiento teórico-conceptual y la incorporación de experiencias de práctica docente que permitan la resolución de problemas de contexto.

\section{Desarrollo profesional de los docentes en servicio}

Avanzar en la consolidación de un sistema de formación profesoral centrado en la promoción de una docencia con excelencia académica y didáctica que responda con pertinencia a las demandas de la sociedad y especialmente de los estudiantes y los contextos en los que se desenvuelven, es un imperativo político y social de un estado que se enfoca en mejorar la calidad de la educación. Este proceso supone no solo el mejoramiento curricular de los programas de formación inicial docente, sino también, el diseño de un plan de actualización de educadores en servicio, que haga frente a los vertiginosos cambios que se producen en la so- 
ciedad como resultado de transformaciones a los sistemas sociales, económicos, políticos, culturales, tecnológicos y científicos.

Por su parte, Tedesco (1999) sostiene que "el análisis del rol de los docentes en este momento de profundos cambios sociales, pone de manifiesto la enorme complejidad de los problemas y la necesidad de enfrentarlos con estrategias sistémicas de acción y no con políticas parciales" (p.23). Esto implica meditar que los docentes considerando su población, la pluralidad de sus perfiles, la diversidad de los contextos en los que se desenvuelven e incluso los niveles en los que se desempeñan, constituyen una realidad altamente compleja que dificulta la construcción de un plan de formación centrado en la actualización y desarrollo profesional de todos los docentes que hacen parte del sistema educativo de un estado a fin de responder con pertinencia a las demandas y necesidades de cada contexto.

Se puede agregar desde esta misma perspectiva que la "formación de los docentes ha de incorporar como un eje fundamental y transversal la investigación y reflexión sobre su práctica, especialmente en lo referido a la didáctica de las diferentes áreas curriculares y a los factores que facilitan el aprendizaje" (UNESCO, 2002, p.23). Este aspecto hace más complicado el cuadro que deben analizar los reformistas de políticas públicas en un tema álgido para los programas de mejoramiento de la calidad educativa como lo es el desarrollo profesional y la actualización de los docentes en ejercicio.

Es ineludible reconocer el trabajo de los educadores como una profesión aun cuando a menudo se le asignen características de una ocupación laboral que en muchos casos no logra producir innovación y que se deja llevar por la cotidianidad. En este sentido la Secretaría de
Educación Pública (2004) sostiene que:

Limitar la enseñanza a una actividad estrictamente técnica e instrumental, tiene un riesgo enorme para el desempeño docente y para el futuro educativo de la nación: significa ignorar su complejidad y reducir las posibilidades de construir una educación de calidad para los niños y los jóvenes. (p.19).

En definitiva, el reto es diseñar un plan de formación docente que pueda responder a las exigencias de los cambios y transformaciones que se han producido en las estructuras sociales, económicas, políticas, culturales, científicas y tecnológicas de contextos regionales y de la realidad universal visible en los procesos de globalización. Esto significa que se requiere de un docente abierto a las nuevas dinámicas de una sociedad que está en permanente evolución y que demanda de ciudadanos que puedan aportar en los nuevos procesos de socialización y desarrollo que se promueven desde los estados y desde las mismas necesidades que se van presentando en los diferentes campos.

En este sentido, es primordial reconocer que "el proceso de formación debe estar organizado para que la capacitación continua o permanente no sea un fragmento de la formación, sino una continuación de la formación inicial" (Lopes, 2012, p.6). Este requerimiento exige un sistema de alineación profesional docente que articule los currículos de profesionalización de las facultades de educación de las universidades con los equipos de direccionamiento que definen la ruta de actualización y desarrollo profesional para los docentes en ejercicio. Como se ha dicho el reto para las políticas educativas es materializar la articulación de un sistema de formación profesoral que logre afianzar la carrera docente, y los planes estratégicos de actualización y desarrollo profesional de manera coherente con las demandas del contexto. 
Vale destacar que en esta instancia resulta relevante emprender la búsqueda de la articulación de las políticas que se están desarrollando en los sistemas de formación docente en la que según Tedesco (1999) "el dominio satisfactorio de la materia a enseñar es una condición necesaria para una enseñanza eficaz, de la misma manera que lo es un dominio satisfactorio de diversas estrategias pedagógicas, que permitan satisfacer las necesidades de una población escolar”. (p.13). En consecuencia, el desafío de mayor envergadura para los sistemas de formación docente es lograr un equilibrio entre el empoderamiento de la disciplina, y el manejo de estrategias didácticas y pedagógicas que favorezcan los aprendizajes de los estudiantes.

En esta perspectiva se destacan experiencias asociadas al acompañamiento pedagógico por parte de pares académicos en los que se promueven escenarios para la planeación, observación de prácticas y reflexión sobre los procesos desarrollados por las y los educadores.

El acompañamiento pedagógico en el aula, en el marco de las estrategias de desarrollo profesional de los docentes en ejercicio, se constituye en una práctica reflexiva e investigativa que tiene como objeto de estudio el aula y los procesos que se desarrollan en la misma. En tal sentido Maureria (2008) sostiene que este:

Permite reflexionar sobre las modalidades de preparación de clases, el uso de estrategias didácticas y metodológicas, el cuidado por el tiempo de trabajo en el aula, así como el manejo de las relaciones entre profesores y alumnos, y entre los estudiantes para velar por el proceso educativo en marcha (p.7).

Lo anterior permite sustentar que el acompañamiento pedagógico se constituye en oportunidad para avanzar en una propuesta de desarrollo profesional docente que prioriza la interacción peda- gógica entre pares académicos, en favor de propiciar ambientes de aprendizaje donde se reflexiona sobre el uso de materiales educativos, distribución del tiempo, didácticas empleadas, tiempo de intervención expositiva del docente, espacios para la participación de los estudiantes, entre otros elementos de gestión de aula.

En este sentido, se puede señalar que el acompañamiento pedagógico es una estrategia que permite la actualización profesional continua del docente, incidiendo en la mejora de su práctica pedagógica y en el desarrollo de habilidades específicas para la orientación de los procesos de enseñanza y aprendizaje en las escuelas. Esto en concordancia con Cardemil, Maureira y Zuleta (2010) quienes sustentan que:

Lograr un mejoramiento escolar significativo requiere de manera indispensable elevar la calidad de las prácticas docentes, para lo cual resulta ineludible acompañar los procesos de aula, acompañamiento que implica observar, luego reflexionar en torno a lo observado y tomar decisiones y acuerdos de mejoramiento (p.1)

En relación al acompañamiento pedagógico Vezub (2011) señala que, “al ser acompañados en su lugar de trabajo, los docentes pueden revisar y apropiarse de los aprendizajes realizados en instancias previas o actuales de su formación, para articularlos con los desafíos que enfrenta su tarea en instituciones y contextos singulares" (p.107). Así mismo, Maureria (2008) destaca "el acompañamiento en la sala de clases para apoyar la aplicación de los nuevos conocimientos y el diálogo profesional para el afianzamiento de la nueva concepción de enseñanza que implementará el profesor". (p.8). En este sentido, el acompañamiento en el aula se transforma en instancia pedagógica para la reflexión sobre los procesos de aprendizaje, y sobre la resignificación de los elementos de la 
gestión de aula por parte de docentes.

En esta misma perspectiva Vezub (2011) concluye que "el acompañamiento proporciona una mediación, una serie de andamiajes y la colaboración necesaria para que los profesores asuman riesgos, animándose a transformar y a enriquecer el trabajo del aula”. (p.107). Teniendo en cuenta estas posturas teóricas se puede señalar que el acompañamiento pedagógico es una estrategia de actualización y desarrollo profesional que logra desde la experiencia del trabajo situado promover los procesos de reflexión y metacognición entre pares académicos, potenciando las competencias pedagógicas para conseguir mejores ambientes de enseñanza y aprendizaje.

Desde otra óptica es relevante considerar la aparición de nuevos escenarios para la formación y el desarrollo profesional de los educadores, gestionados por los avances de las nuevas tecnologías de la información y la comunicación, favoreciendo el tránsito de una mediación pedagógica presencial a una mediación tecnológica que utiliza como interfaz el ordenador y la conectividad. Sin embargo, en la actualidad pese a los avances que se han dado en los sistemas educativos, continúan siendo significativas las brechas entre regiones desarrolladas y lugares de difícil acceso donde no se cuenta con este tipo de herramientas. Las distancias para el acceso de los docentes como el acceso de los estudiantes siguen siendo significativas.

Frente al asunto de las tecnologías de la información y la comunicación en el contexto de la formación y desarrollo profesional de los docentes Tedesco (1999) señala la necesidad de "desmitificar el secreto que rodea la utilización de estas tecnologías, así como diseñar políticas democráticas que permitan un acceso equitativo a su conocimiento y utilización es una exigencia impostergable de toda estrategia destinada a fortalecer el rol de los docentes". (p.22). Frente a este tema es relevante considerar a Swig (2011) quien sostiene que "los maestros que ingresan a la fuerza laboral no están adecuadamente preparados para utilizar las Tecnologías de la Información y la Comunicación como recursos para enseñar y mejorar el aprendizaje de los estudiantes". (p.2) En consecuencia se hace necesario que los sistemas de actualización y desarrollo profesional de docentes en ejercicio diseñen estrategias contextualizadas en las posibilidades de las escuelas y sus entornos que posibiliten el desarrollo de competencias tecnológicas y pedagógicas para el aprovechamiento de herramientas y ambientes ofrecidos por las Nuevas Tecnologías de la Información y la comunicación en los procesos de enseñanza-aprendizaje.

Por su parte, Boer (2014) afirma que "la escuela necesita, poner en primer lugar las necesidades de aprendizaje de las nuevas generaciones, pensar en un futuro que es muy difícil imaginar y entregarles herramientas para desenvolverse en él”. (p.22) En esta apuesta el uso adecuado de las tecnologías de la información y la comunicación juegan un papel preponderante en la medida en que permiten el desarrollo de nuevas formas de mediación didáctica en ambientes dinámicos e interactivos en los que generalmente los estudiantes se sienten motivados.

Los estudios sobre este tema revelan que "las tutorías entre pares son una técnica de clase relativamente común en diversas culturas alrededor del mundo. Este modelo es efectivo incluso cuando los docentes tienen un conocimiento muy limitado de las TICs". (Unesco, 2004, p.42). El reto para los reformistas de políticas educativas de desarrollo profesional docente es precisamente la proyección de estrategias que permitan la formación situada entre pares académicos, considerando las necesidades reales que experimentan los estudiantes en sus contextos y con las herramientas tecnológicas disponibles en los centros escolares.

En este contexto si "una escuela en su conjunto -docentes, equipos directivos, 
administradores - no trabaja como equipo para incorporar la tecnología en los sistemas de educación, un docente individual no será capaz de tener éxito en el uso de las tecnologías en sus clases" (Swig, 2011, p.). Esta situación exige que las organizaciones educativas en su direccionamiento estratégico logren la consolidación de comunidades de aprendizaje como mecanismo para el desarrollo de los procesos de planeación y puesta en escena de las intervenciones pedagógicas en el aula de clase.

\section{Conclusiones}

La profesionalización y formación inicial de los educadores es uno de los temas de mayor relevancia en las agendas de política pública de los estados en su apuesta por mejorar la calidad de la educación. En este sentido se reconoce que en algunos casos el sistema de formación inicial ha sido insuficiente para proporcionar a los futuros educadores los conocimientos, generales y específicos, y los elementos pedagógicos, didácticos y científicos fundamentales para el ejercicio profesional de la tarea docente.

Los nuevos escenarios han producido diversas demandas sociales para el sistema educativo y en particular para los diseños curriculares de los programas de formación docente en lo que se refiere a contenidos de enseñanza y modelos didácticos empleados en las instituciones educativas, acarreando consigo la necesidad de redefinir el rol del docente en los procesos de enseñanza y aprendizaje. En consecuencia la formación que se propende desde las mallas curriculares de los programas de formación inicial docente no es coherente con la realidad social en que se desarrollan los estudiantes. Esto trae consigo que las nuevas demandas a nivel de estructuras sociales, económicas, culturales y políticas conllevan a asumir un cambio de perspectiva, nuevos estilos de enseñanza, en un marco de permanente revisión y cuestionamiento.

Se determina que los educadores del nivel de educación básica primaria requieren tener una mejor formación en el campo disciplinario, mientras que los docentes de educación media necesitan fortalecer sus competencias didácticopedagógicas para el abordaje en el aula de los saberes disciplinares.

Con respecto a los programas de formación docente que ofertan las escuelas normales y las universidades se resalta el marcado interés curricular en el desarrollo conceptual y teórico de las disciplinas, y la necesidad de fortalecer los espacios para la práctica pedagógica que le permita a los futuros docentes el desarrollo de competencias funcionales y comportamentales básicas para el óptimo desempeño en los procesos de enseñanza aprendizaje.

Finalmente, en lo referente a la actualización y desarrollo profesional de docentes en ejercicio se destaca la complejidad de dicho sistema debido a la pluralidad de sus perfiles, la diversidad de los contextos en los que se desenvuelven e incluso los niveles en los que se desempeñan. Estos elementos constituyen una realidad compleja que dificulta la construcción de un plan de formación centrado en la actualización y desarrollo profesional de todos los docentes que hacen parte del sistema educativo de un estado a fin de responder con pertinencia a las demandas y necesidades de cada contexto.

El reto en esta área es diseñar un plan de formación docente que responda con pertinencia a las exigencias de las transformaciones que se han promovido como consecuencia de los procesos de globalización. Esto implica la construcción participativa de un sistema de alineación profesional docente que articule los currículos de profesionalización de las facultades de educación de las universidades y de las Escuelas Normales con los equipos de direccionamiento que trazan la ruta de actualización y desarrollo profesional para los docentes en ejercicio. 


\section{Referencias}

Aguerrondo, I (2003). Formación docente: desafíos de la política educativa. Maestros en América Latina, 98-141. Recuperado de:

http://www.oei.es/historico/docentes/articulos/desafios_politica_educativa_reformas_formacion_ docente_aguerrondo.pdf

Boer, I. (2014). El rol de los profesores está cambiando, su formación y desarrollo profesional también. Mirada RELPE Reflexiones iberoamericanas sobre las TIC y la educación, volumen (1). 21-25. Recuperado de: www.relpe.org/descargas/miradarelpe.pdf

Cardemil, C., Maureira, F. y Zuleta, J. (2010). Modalidades de acompañamiento y apoyo pedagógico al aula. 1-3. Recuperado de: http://mailing.uahurtado.cl/cuaderno_educacion_31/pdf/instrumento31.pdf

Cornejo, J. (2014). Prácticas profesionales durante la formación inicial docente: análisis y optimización de sus aportes a los que aprenden y a los que enseñan a aprender "a enseñar". Estudios Pedagógicos, volumen (40). 239-256. Recuperado de: http:// www.scielo.cl/scielo.php?script=sci_arttext\&pid $=$ S0718-07052014000200014

Correa, E. (2011). La práctica docente una oportunidad de desarrollo profesional. Perspectiva educacional, volumen (50), 77-95. Recuperado de: http://www. perspectivaeducacional.cl/index.php/peducacional/article/viewFile/41/21

Imbernon, F. (1989). La formación inicial y la formación permanente del profesorado. Dos etapas de un mismo proceso. Revista interuniversitaria de formación del profesorado (6), 487-499. Recuperado de: file://C:/Users/User\%20Compumax/ Downloads/Dialnet-LaFormacionInicialYLaFormacionPermanenteDelProfeso-117680.pdf

Internacional de la Educación Oficina Regional para América Latina. (2014). La Formación Docente en américa Latina (1). Recuperado de: http:// www.ei-ie-al.org/publicaciones/formacion-esppor_web.pdf

Lopes, L.M. (2012). La formación inicial de las educadoras y de los educadores: profesores y profesoras. Frizona freireano, volumen (12), 1-18. Recuperado de: http://www.rizoma-freireano.org/index. php/la-formacion-inicial-de-las-educadores-yde-los-educadores-profesores-y-profesoras-lenamaria-pires-coreia-lopes-marcal

Maureria, F. (2008). Estrategia de Acompañamiento a Establecimientos Educativos vulnerables, 1-10. Recuperado de: http://creasfile.uahurtado.cl/aco mpa\%C3\%B1amientoeducacionalesvulnerables. pdf

Mercado, R. (2010). Un debate actual sobre la formación inicial de docentes en México. Revista Semestral da Associação Brasileira de Psicología Escolar e Educacional, SP, volumen (14), 149-157. Recuperado de: http://www.scielo.br/pdf/pee/v14n1/ v14n1a16.pdf

Murillo, F.J. (2006). Panorámica general de las aportaciones innovadoras. Modelos innovadores en la formación inicial docente, 17-54. Recuperado de:

https://web.oas.org/childhood/ES/Lists/Recursos\%20 \%20Bibliografia/Attachments/85/96.pdf

Paniagua, M. (2004). La formación y la actualización de los docentes: herramientas para el cambio en educación. Procesos de aprendizaje, 1-18. Recuperado de: http://www.cedal.org/docus/educ01.pdf

Pogré, P. y Merodo, A. (2006). Un modelo multidisciplinar en la formación de profesores. Modelos innovadores en la formación inicial docente, 57-110. Recuperado de: https://web.oas.org/childhood/ ES/Lists/Recursos\%20\%20Bibliografia/.../85/96. pdf

Rodríguez, V. (2014). La formación situada y los principios pedagógicos de la planificación: la secuencia didáctica. Ra Ximhai, volumen 10 (5), 323331. Recuperado de: http://uaimlosmochis.org/ ECFD/index.php/2014/2/paper/viewFile/223/38

Secretaría de Educación Pública. (2004). Hacia una 
política integral para la formación y el desarrollo profesional de los maestros de educación básica. Documento Base. Cuadernos de discusión 1. Recuperado de:

http://wbgfiles.worldbank.org/documents/hdn/ed/ saber/supporting_doc/LCR/Teachers/Mexico/ AS_S6_MEXICO_BaseDocument-PolicyofTeacherDevelopment_03.pdf.

Swig, C. (2015). TICs y formación docente: formación inicial y desarrollo profesional docente. Notas de Política PREAL, 1-8. Recuperado en: https://www.oas.org/cotep/GetAttach. aspx?lang=En\&cId=265\&aid $=417$

Tedesco, J.C. (1999). Profesionalización y capacitación docente. El Instituto Internacional de Planeamiento de la Educación, Unesco, 3-23. Recuperado de: http://www.buenosaires.iipe.unesco.org/documentos/profesionalizaci-n-y-capacitaci-n-docente

UNESCO (2002). Proyecto Regional de Educación para América Latina y el Caribe. Modelo de acompañamiento -apoyo, monitoreo y evaluación- para América latina y el caribe. Declaración de la Habana. Recuperado de: http://unesdoc.unesco.org/ images/0013/001316/131687so.pdf
UNESCO (2004). Las tecnologías de la información y la comunicación en la formación docente. Guía de planificación. Recuperado en: http://unesdoc. unesco.org/images/0012/001295/129533s.pdf

Universidad de la Salle. (2010). La formación y el desarrollo profesional docente. Documentos Institucionales No 37. Recuperado en: http://www. lasalle.edu.co/wps/wcm/connect/cd3bd6fe48ac-41e2-9bef-bbb2e0212438/Librillo_37. pdf?MOD=AJPERES

Vaillant, D. (2013). Formación Inicial del profesorado en América Latina: Dilemas centrales y perspectivas. Revista Española de Educación Comparada, volumen (22), 185-206. Recuperado en: http://denisevaillant.net/articulos/2013/Formacion_inicial_profesorado_America_Latina.pdf (diciembre 15 de 2016).

Vezub, L. (2011). Las políticas de acompañamiento pedagógico como estrategia de desarrollo profesional docente. Revista del IICE, volumen (30), 103124. Recuperado en: http://revistascientificas.filo. uba.ar/index.php/iice/article/view/149/111 